\title{
Performance of SuperSTAT Method of Non Invasive Blood Pressure (NIBP) Measurement Technique at Different Inflation Levels
}

\author{
Prakash Dirshanapu \\ Department of ECE M Tech, National Institute of \\ Technology Warangal
}

\begin{abstract}
The purpose of this paper is to analyze the performance of oscillometric based super STAT method which is used to determine automatic Non Invasive Blood Pressure (NIBP). Mainly four target cuff pressures are chosen in the range of $115 \mathrm{mmHg}$ to $160 \mathrm{mmHg}$ and at each target cuff pressure the NIBP parameters are measured from 10 numbers of subjects which are performed on left and rights hands and recorded. Eventually the correlation graph is plotted for systolic, diastolic, MAP (Mean Arterial pressure) and pulse readings using statistical analysis tools. The results of the correlation plot states that systolic, diastolic and MAP are moderately correlated at low target inflation cuff pressure and in the range of $135 \mathrm{mmHg}$ to $160 \mathrm{mmHg}$ cuff inflation pressure the correlation is high between left and right hand NIBP determinations. Pulse measurements are highly correlated at all the cuff inflation levels. Thus the super STAT algorithm gains better accuracy over basic oscillometric method of NIBP determination.
\end{abstract}

\section{General Terms}

BP Determination, Correlation, MAP, NIBP, Oscillometric method, pulse

\section{Keywords}

Accuracy, Cuff, Diastolic, NIBP, Super STAT Target Inflation, Systolic

\section{INTRODUCTION}

Since many years, noninvasive blood pressure (NIBP) monitors have been extensively used in operating rooms and condemnatory care units to closely monitor blood pressure in patients of all the age groups. This parameter defines systemic circulation or arterial blood pressure which is used for predict function of heart, pathology of blood vessel, tissue perfusion, organ perfusion and function of kidney. Most conventional medical devices used at health center are the automatic noninvasive blood-pressure (NIBP) meter .A cuff is encased around the arm of the patient and inflated upto certain level to determine NIBP. Most of the automatic blood-pressure meters are built on the oscillometric principle, which registers the oscillations in the cuff pressure during the cuff deflation and thus systolic, diastolic, MAP (Mean Arterial pressure) and pulse are determined using a mathematical algorithm. The MAP can be defined as mean arterial pressure during a mono cardiac cycle and sometimes computed using systolic and diastolic readings. Despite the extensive use of automated blood pressure monitors, clinicians continue to conscious over the accuracy and fidelity of automated NIBP devices compared to other methods of blood pressure determination.

\author{
Jatoth Ravi Kumar \\ Department of ECE, Associate Professor, National \\ Institute of Technology Warangal, India
}

Today's generation has many types of NIBP measurements techniques and SuperSTAT is one of those.

\subsection{SuperSTAT NIBP Determination}

The oscillometric method of determining SuperSTAT NIBP is achieved by a sensitive transducer which calculates the cuff pressure and tiny pressure oscillations within the cuff. The first determination initially pumps up to an initial target cuff pressure of about $135 \mathrm{mmHg}$ or to the custom initial target pressure.

The oscillations versus cuff pressure are measured, and ultimately, systolic, mean, and diastolic pressures are determined. When establishing fewer pressure steps, the system will make use of stored information from the prior blood pressure determination to determine the best pressure steps to take. If the current blood pressure reading is common to the previous reading, the system may use some information from the previous blood pressure in the current determination. This technique of determining the NIBP permits to set the initial inflation pressure to a definite value which is decided optimally by diagnoses. There were many research done to find the correlation between oscillometric and auscultation methods and concluded the auscultation is a golden method for NIBP. . The previous research, Yasemin G. (2008) presented the comparing result of auscultation and oscillometric method that was high correlation and oscillometric used easily more than auscultation [1]. ShinSihin Chio (2011) stated the data that SBP of oscillometric is higher than SBP of auscultation [2].

In term of DBP, Oscillomatric was lower than auscultation. Mohamad Forouzanfar (2014) states about empirical ratios on the oscillometric waveform envelope (OMWE) to determine the systolic pressure (SP) and diastolic pressure (DP) which is employed in most of the oscillometric based NIBP methods [3]. H.D. Kiers (2008) compared MAP of both methods that ware non significance [4]. Bur A (2000) concluded that The relation between cuff size and upper-arm circumference contributes substantially to the inaccuracy of the oscillometric blood pressure measurement [5]. Pediatrics and Adolescent Medicine (2001) reported the blood pressure measurement by auscultation method and oscillometric method that ware different value. Here in this paper only a SuperSTAT method of NIBP measurement is considered to find the left and right hand NIBP correlation at different target cuff pressure or target inflation levels. Figure 1 shows the basic oscillometric method of measuring NIBP. 


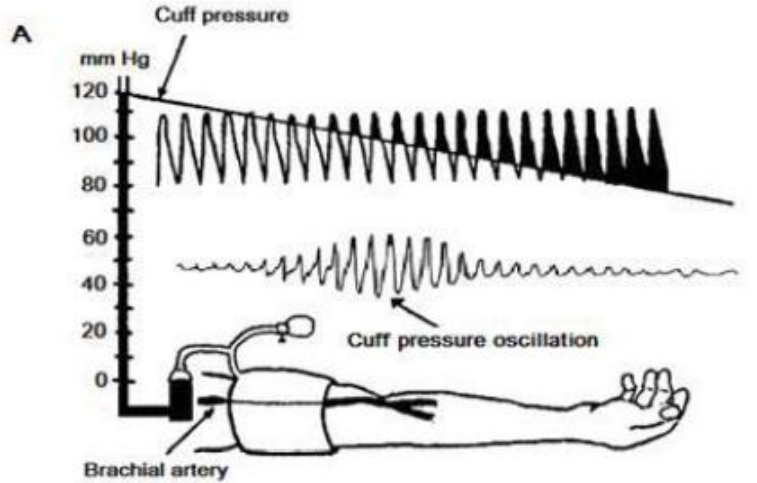

Figure 1: Oscillometric method of NIBP measurement

\section{PERFORMANCE ANALYSIS TEST}

\subsection{Subject}

A 10 number of hygienic subjects without any cardiovascular disease had gone through NIBP test at Four inflation levels say $115 \mathrm{mmHg}, \quad 135 \mathrm{mmHg} 155 \mathrm{mmHg}$ and $160 \mathrm{mmHg}$ respectively. All the subjects are males and aged between 21 and 35. As this test is a general NIBP reading, no ethical permission or consent is needed.

\subsection{Test Procedure}

There are various possible ways of examine the performance of super STAT algorithm which is used in Health Care Commercial product. In this paper we adopted a method which deals with four inflation levels say $115 \mathrm{mmHg}$, $135 \mathrm{mmHg}, 155 \mathrm{mmHg}$ and $160 \mathrm{mmHg}$ respectively. The tests are performed on right and left hands of every subject and NIBP readings are recorded.

The test procedure is as follows:

1) Initially the inflation level is set $115 \mathrm{mmHg}$ using user access keys.

2) Every volunteer/subject is allowed to sit on a chair and the arm is placed at heart level before measuring.

3) The appropriate cuff is selected for the volunteer based on age and arm size (in this test all are adult cuffs as all the subjects are adult category).
4) The cuff is wrapped properly on right hand allowing a finger gap so that the fault readings are avoided.

5) Systolic, diastolic, MAP (Mean Arterial pressure) and pulse values are obtained on the setup screen by accessing the single NIBP button.

6) Steps 2, 3 and 4 are repeated on left hand as performed on right hand.

7) Again the steps 3 and 4 are repeated for $135 \mathrm{mmHg}$, $155 \mathrm{mmHg}$ and $160 \mathrm{mmHg}$ inflation levels.

8) For each and every measurement a time interval of $5 \mathrm{~min}$ is provided for arterial relaxation so that no erroneous readings come into picture.

Table 1: General information of subjects

\begin{tabular}{|l|rl|l|}
\hline S.No & Information & Percentage \\
\hline 1 & Gender & $100 \%$ \\
& $\bullet \quad$ Male & $0 \%$ \\
\hline 2 & Age $\quad$ Female & $60 \%$ \\
& $\bullet \quad 21-25 y r s$ & $30 \%$ \\
& $\bullet \quad 26-30 y r s$ & $10 \%$ \\
& $\bullet \quad$ Yes & $80 \%$ \\
\hline 3 & Exercise & $20 \%$ \\
& $\bullet \quad$ No & 173.6 \\
\hline 4 & Mean height of subjects & 72.55 \\
\hline 5 & Mean weight of subjects & \\
\hline
\end{tabular}



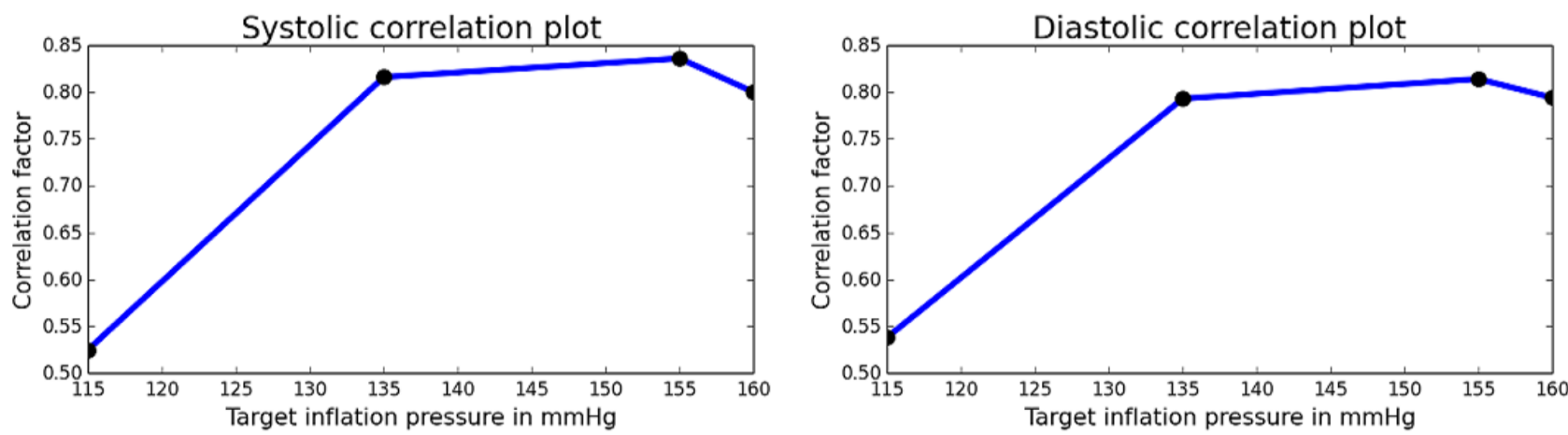

MAP correlation plot
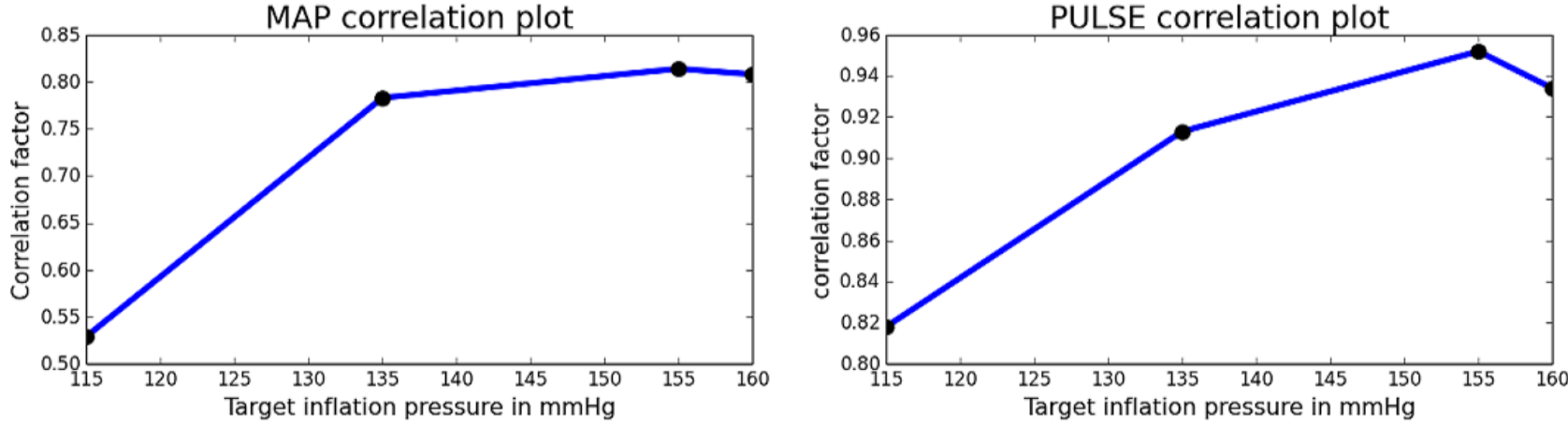

Figure 2: Correlation Plot

\section{CORRELATION PLOT}

The correlation plots for the Systolic diastolic, MAP and pulse readings which are obtained from above procedure is manipulated using statistical analysis tool and are shown below The mean values of systolic, diastolic, MAP and Pulse for right and left hands combining all inflation levels are given in Table 2.

Table 2: NIBP parameters mean values

\begin{tabular}{|l|l|l|l|}
\hline S.no & NIBP Parameters & \multicolumn{2}{|l|}{ Mean Value } \\
\cline { 3 - 4 } & & Right Hand & $\begin{array}{l}\text { Left } \\
\text { Hand }\end{array}$ \\
\hline 1 & Systolic $(\mathrm{mmHg})$ & 115.14 & 116.03 \\
\hline 2 & Diastolic $(\mathrm{mmHg})$ & 72.46 & 74.22 \\
\hline 3 & MAP $(\mathrm{mmHg})$ & 87.50 & 88.23 \\
\hline 4 & Pulse $(\mathrm{mmHg})$ & 72.94 & 73.68 \\
\hline
\end{tabular}

Table 3: Correlation coefficients of NIBP parameters

\begin{tabular}{|l|l|l|l|l|l|}
\hline S.no & $\begin{array}{l}\text { NIBP } \\
\text { Parameter } \\
\text { s }\end{array}$ & \multicolumn{4}{|l|}{ Target cuff pressure $(\mathrm{mmHg})$} \\
\cline { 3 - 6 } & $\begin{array}{l}\text { Systolic( } \\
\text { mmHg) }\end{array}$ & 0.525 & 0.816 & 0.836 & 0.801 \\
\hline 2 & $\begin{array}{l}\text { Diastolic( } \\
\text { mmHg) }\end{array}$ & 0.538 & 0.793 & 0.814 & 0.794 \\
\hline 3 & $\begin{array}{l}\text { MAP } \\
(\mathrm{mmHg})\end{array}$ & 0.529 & 0.783 & 0.814 & 0.808 \\
\hline 4 & $\begin{array}{l}\text { Pulse(mm } \\
\mathrm{Hg})\end{array}$ & 0.818 & 0.913 & 0.952 & 0.934 \\
\hline
\end{tabular}

Table 3 shows the correlation coefficients of NIBP parameters. The correlation factor for the Blood Pressure parameters at different target inflation levels calculated for both the arms, states the closeness of determinations and the above plots defines the correlation coefficient variation at mentioned inflation levels. These parameters are strongly correlated for the cuff inflation levels 135,155 and $160 \mathrm{mmHg}$ but correlated moderately at $115 \mathrm{mmHg}$. The target cuff pressure has to be selected based on patient category with appropriate cuff selection. Violation of these steps leads to erroneous readings.

\section{CONCLUSIONS}

There are many methods for NIBP measurement based on oscillometric principle and most automatic NIBP medical units implement it.Super STAT algorithm is also a based on oscillometric method and has better accuracy over the basic oscillometric methods and this technique of measuring the NIBP allows to set the initial inflation pressure to a certain value which is best decided by diagnoses. From the above plots we can observe that while selecting a lower inflation levels for adult subjects the correlation is moderate.

It is not mandatory to inflate higher cuff pressure to adult patients because it can be best decided by diagnoses based on health condition and totally a medical concern. However if the target cuff pressure is less than the systolic pressure, the super STAT algorithm is capable of inflating the cuff pressure over the systolic and produce NIBP automatically. Eventually this paper defines that selecting a suitable cuff pressure will produce more accurate readings.

\section{REFERENCES}

[1] Genc Yasemin, Altunkan Sekip, Kiline Oguzhan, Altunkan Erkan. Comparative study on auscultatory and oscillometric methods of ambulatory blood pressure measurements in adult patients Blood Pressure Monitoring 2008; 13: 29-35. 
[2] Chio Shiu-Shin, Urbina Elaine M., orotkoff sound versus oscillometric cuff sphygmomanometers: comparison between auscultatory and DynaPulse blood pressure measurements. Journal of the American Society of Hypertention 2011; 5(1): 12- 20.

[3] Mohamad Forouzanfar, Hilmi R. Dajani, Voicu Z. Groza, Miodrag Bolic, Sreeraman Rajan, and Izmail BatkinRatio Independent Blood Pressure Estimation by Modeling the Oscillometric Waveform Envelope, VOL. 63, NO. 10, OCTOBER 2014.

[4] Kiers H.D., Hofstra J.M., Wetzels J.F.M., Oscillometric blood pressure measurements differences between measured and calculated mean arterial pressure. The Journal of Medicine 2008; 66(11): 474-479.
[5] Bur A, Hirschl MM, Herkner H, Oschatz E, Kofler J, Woisetschläger C, Laggner AN. Accuracy of oscillometric blood pressure measurement according to the relation between cuff size and upper-arm circumference in critically ill patients, 2000 Feb; 28(2):371-6.

\section{AUTHORS}

First Author- D Prakash, Department of ECE M Tech, National Institute of Technology Warangal

Second Author- Jatoth Ravi Kumar, Department of ECE, Associate Professor, National Institute of Technology Warangal 